\title{
On isoperimetric inequalities with respect to infinite measures
}

\author{
Friedemann Brock, Anna Mercaldo and Maria Rosaria Posteraro
}

\begin{abstract}
We study isoperimetric problems with respect to infinite measures on $\mathbb{R}^{n}$. In the case of the measure $\mu$ defined by $d \mu=e^{c|x|^{2}} d x, c \geq 0$, we prove that, among all sets with given $\mu$-measure, the ball centered at the origin has the smallest (weighted) $\mu$-perimeter. Our results are then applied to obtain Pólya-Szegö-type inequalities, Sobolev embedding theorems, and a comparison result for elliptic boundary value problems.
\end{abstract}

\section{Introduction}

Consider an elliptic boundary value problem of the following type,

$$
\left\{\begin{aligned}
-\operatorname{div}\left(\varphi(x)|\nabla u|^{p-2} \nabla u\right) & =f \varphi(x) & & \text { in } \Omega \\
u & =0 & & \text { on } \partial \Omega,
\end{aligned}\right.
$$

where $\Omega$ is an open subset of $\mathbb{R}^{N}$, possibly unbounded, and $f$ belongs to a suitable weighted Lebesgue space. We are interested in sharp explicit a priori bounds for the weak solution to (1.1). Such problems can be examined by symmetrization methods. However, the presence of the weight function in the operator in (1.1) does not allow us to use the classical approach via Schwarz symmetrization given, e.g., in [30] and [3]. This leads us to introduce an appropriate symmetrization based on a weighted isoperimetric inequality which is related to the structure of the operator. A similar approach which is based on the isoperimetric inequality for the Gauss measure has been carried out by the authors in [5] (see also [14] and $[9])$.

In this paper we study isoperimetric inequalities for infinite measures, together with properties of the corresponding weighted symmetrizations. To be more precise, let $\mu$ be a measure on $\mathbb{R}^{n}$ defined by

$$
d \mu=\varphi(x) d x,
$$

Mathematics Subject Classification (2010): 26D20, 35J70, 46E35.

Keywords: Isoperimetric inequalities, infinite measures, Steiner symmetrization, Schwarz symmetrization, comparison result, Pólya-Szegö inequality. 
where $\varphi$ is a positive continuous function. For any smooth set $\Omega$, we denote by

$$
P_{\mu}(\Omega)=\int_{\partial \Omega} \varphi(x) d \mathcal{H}_{n-1}(x)
$$

the weighted perimeter of $\Omega$ w.r.t. $\mu$, and for any fixed number $m>0$, we denote by $I_{\mu}(m)$ the isoperimetric function, that is

$$
I_{\mu}(m):=\inf \left\{P_{\mu}(\Omega): \Omega \text { smooth, } \mu(\Omega)=m\right\} .
$$

We are interested in finding isoperimetric sets, that is smooth sets which realize the infimum in (1.2).

Such a problem has been treated in various settings. For example, if $\mu$ is the Lebesgue measure on $\mathbb{R}^{n}$, then the isoperimetric sets are the balls, i.e., if $\varphi(x) \equiv 1$, then $I_{\mu}(m)=P_{\mu}(B)$, for any ball $B$ in $\mathbb{R}^{n}$ with $\mu(B)=m$ (see for instance [29] and $[32])$.

Moreover if $\mu$ is the Gauss measure, then the isoperimetric sets are the halfspaces of $\mathbb{R}^{n}$, i.e. if $\varphi(x)=\exp \left(-c|x|^{2}\right)$ for some $c>0$ and $m \in\left(0, \mu\left(\mathbb{R}^{n}\right)\right)$, then $I_{\mu}(m)=P_{\mu}(H)$, where $H$ is any Euclidean half-space with $\mu(H)=m$ (see for instance [7], [15], and [22]).

Isoperimetric inequalities and their connections with rearrangements have received considerable interest in the last decades (see e.g. [20], [10], [31], [26], [27], and the references cited therein). In the paper [6], the authors recently analyzed symmetrizations w.r.t. finite measures on $\mathbb{R}^{n}$.

Here we investigate infinite measures on $\mathbb{R}^{n}$ together with Steiner and Schwarz symmetrizations. One of our results is the following: if $\mu$ is a measure defined by

$$
d \mu=\exp \left(c|x|^{2}\right) d x, \quad \text { for some } c>0,
$$

then the only isoperimetric sets are Euclidean balls which are centered at the origin, i.e., one has

$$
I_{\mu}(m)=P_{\mu}\left(B_{R}\right)
$$

where $R$ is chosen in such a way that $\mu\left(B_{R}\right)=m$. Alternatively one can express this isoperimetric inequality by using the notion of the weighted Schwarz symmetrization $U^{\star}$ of a set $U$, which is the Euclidean ball centered at the origin such that $\mu(U)=\mu\left(U^{\star}\right)$. With this notation, (1.4) is equivalent to

$$
P_{\mu}(U) \geq P_{\mu}\left(U^{\star}\right), \quad \text { for any smooth set } U,
$$

with equality if and only if $U=U^{\star}$ (see Theorem 5.1).

We note that a proof of inequality (1.5), but without the equality case, was given by Borell already in 1986, in an unpublished preprint (see [8]). Theorem 5.1 was the subject of an earlier preprint of the authors, and it has been presented at several conferences since 2005. In 2006 Benguria and Linde in [4] used this result to obtain eigenvalue bounds for the Dirichlet Schrödinger operator.

We emphasize that after having finished our paper we learned that Theorem 4.4 has been also proved independently by Rosales, Cañete, Bayle and Morgan ([28]). 
All the cited proofs are based on the observation that Steiner symmetrization does not increase the perimeter. Note that our proof differs from the one given in [28] in that it does not make use of the smoothness of a minimizing set. Therefore we include it here, for the convenience of the reader. It is performed in several steps.

First we study the one-dimensional case. More precisely, we consider a measure on $\mathbb{R}$ given by

$$
d \mu_{1}=\psi\left(x_{1}\right) d x_{1},
$$

where $\psi$ is an even, positive and continuous function on $\mathbb{R}$ and we prove that (1.4) holds if and only if $\psi$ is log-convex. Then we consider a more general measure $\mu$ whose density is the product of two continuous functions $\psi: \mathbb{R} \rightarrow \mathbb{R}_{+}$, and $\rho$ : $\mathbb{R}^{n-1} \rightarrow \mathbb{R}_{+}$depending on $x_{1}$ and $x^{\prime}=\left(x_{2}, \ldots, x_{n}\right)$ respectively, i.e.,

$$
d \mu=\psi\left(x_{1}\right) \rho\left(x^{\prime}\right) d x
$$

we prove that Steiner symmetrization with respect to this measure decreases the perimeter (see Section 4 for the definition of Steiner symmetrization). The last step consists in approximating the ball $U^{\star}$ by an appropriate sequence of successive Steiner symmetrizations. In view of the product structure of the density and the invariance w.r.t. rotations of the measure (1.3), this leads to (1.5).

Isoperimetric inequality (1.5) has various consequences. For example, by Talenti's result ([31]), inequality (1.5) implies a Pólya-Szegö type inequality (see Theorem 5.4) and the equality case is also studied. Moreover, we prove a Sobolev type imbedding theorem in a weighted space w.r.t. the measure $\mu$ defined in (1.3) (see Theorem 5.5). The best constant in such an inequality is obtained in a special case (see Corollary 5.7).

We now outline the content of the paper. In Section 2 we introduce some notation and provide basic information about the weighted perimeter. In Section 3 we study the symmetrization w.r.t. general measures, on the real line. In Section 4 we prove isoperimetric inequalities w.r.t. product measures in $\mathbb{R}^{N}$. In particular, we show that the Steiner symmetrization decreases the weighted perimeter of a set having given measure. We also prove general Pólya-Szegö-type inequalities (see Theorem 4.12). In Section 5 we study measures whose densities are radially symmetric, and in particular we show inequality (1.5). Finally, Section 6 contains the comparison result; a similar result is given in [6].

\section{Notation and preliminaries}

In the whole paper $\mu$ will denote a measure on $\mathbb{R}^{n}$ defined by

$$
d \mu=\varphi(x) d x
$$

where $\varphi \in C\left(\mathbb{R}^{n}\right)$ and $\varphi(x)>0$ for any $x \in \mathbb{R}^{n}$. Moreover $\mu_{1}$ will denote a measure on $\mathbb{R}$ defined by

$$
d \mu_{1}=\psi(x) d x
$$

where $\psi \in C(\mathbb{R})$ and $\psi(x)>0$ for any $x \in \mathbb{R}$. 
We will always assume that the measures are infinite, that is

$$
\mu\left(\mathbb{R}^{n}\right)=+\infty, \quad \mu_{1}(\mathbb{R})=+\infty .
$$

By $\mathcal{G} M^{n}$ we denote the set of $\mu$-measurable sets with finite Lebesgue measure and by $\mathcal{F}^{n}$ the set of all $\mu$-measurable functions on $\mathbb{R}^{n}$ such that $\{x: u(x)>t\} \in \mathcal{M}^{n}$ for every $t>\inf u$.

Let $\Omega$ be a domain in $\mathbb{R}^{n}$ and $p \in[1,+\infty)$. We denote by $L^{p}(\varphi, \Omega)$ the space of $\mu$-measurable functions $u$ such that

$$
\|u\|_{p, \Omega} \equiv\left(\int_{\Omega}|u|^{p} d \mu\right)^{1 / p}<+\infty
$$

endowed with the norm (2.4). Furthermore, let $W^{1, p}(\varphi, \Omega)$ denote the weighted Sobolev space containing all functions $u \in L^{p}(\varphi, \Omega)$ with weak derivatives $u_{x_{i}} \in$ $L^{p}(\varphi, \Omega), i=1, \ldots, n$, and let

$$
|\|u\||_{p, \Omega}:=\|u\|_{p, \Omega}+\|\nabla u\|_{p, \Omega}
$$

be a norm in this space. Finally, let $W_{0}^{1, p}(\varphi, \Omega)$ be the closure of $C_{0}^{\infty}(\Omega)$ under the norm (2.5). If $\Omega=\mathbb{R}^{n}$ in one of the above spaces, then we will omit the subindex $\Omega$ in the norms.

For subsets $A, B, M$ of $\mathbb{R}^{n}$, let $A+B=\{x+y: x \in A, y \in B\}$ denote the Minkowski sum of $A$ and $B$, and

$$
M_{r}:=\left\{x \in \mathbb{R}^{n}: \operatorname{dist}\{x ; M\}<r\right\}=M+B_{r}, \quad r>0,
$$

the exterior parallel sets of $M$, where $B_{r}$ is the ball of radius $r$ with the center at the origin.

We will call a set $M \subset \mathbb{R}^{n}$ smooth if $M$ is bounded and open, and if there is a number $\varepsilon>0$ such that for every $x_{0} \in \partial M, \partial M \cap B_{\varepsilon}\left(x_{0}\right)$ is a Lipschitz graph, and $M \cap B_{\varepsilon}\left(x_{0}\right)$ lies on one side of $\partial M \cap B_{\varepsilon}\left(x_{0}\right)$. Observe that this definition includes polyhedra and excludes the presence of "veils" and inner "slices" of $M$.

If $M$ is a Borel set then we denote by $\mu^{+}(M)$ the (lower outer) Minkowski $\mu$-content of the boundary of $M$ (see, for instance, [10], p. 69) which is defined by

$$
\mu^{+}(M)=\liminf _{r \rightarrow 0^{+}} \frac{\mu\left(M_{r}\right)-\mu(M)}{r} .
$$

If $\varphi \in W_{l o c}^{1,1}\left(\mathbb{R}^{n}\right)$, then we define the $\mu$-perimeter (in the sense of De Giorgi) by

$$
P_{\mu}(M)=\sup \left\{\int_{M} \operatorname{div}(\varphi(x) h(x)) d x: h \in C_{0}^{1}\left(\mathbb{R}^{n}, \mathbb{R}^{n}\right),|h| \leq 1\right\} .
$$

The following properties are well known for the Lebesgue measure (see, for instance, [10]) and their proofs carry over to general measures $\mu$ defined in (2.1):

1) Both $\mu^{+}(M)$ and $P_{\mu}(M)$ can be seen as "weighted surface measures" of $M$, that is, if $M$ is a smooth set then

$$
\mu^{+}(M)=P_{\mu}(M)=\int_{\partial M} \varphi(x) \mathcal{H}_{n-1}(d x)<+\infty .
$$


2) Lower semicontinuity: If $\left\{M_{k}\right\} \subset \mathcal{M}^{n}, M \in \mathcal{M}^{n}$, and $\mu\left(M \Delta M_{k}\right\} \rightarrow 0$ as $k \rightarrow \infty$, then

$$
\liminf _{k \rightarrow \infty} P_{\mu}\left(M_{k}\right) \geq P_{\mu}(M) .
$$

3) If $M \in \mathcal{M}^{n}$, and $P_{\mu}(M)<\infty$, then there is a sequence of smooth sets $\left\{M_{k}\right\}$ such that $\mu\left(M \Delta M_{k}\right) \rightarrow 0$ as $k \rightarrow \infty$, and such that

$$
\lim _{k \rightarrow \infty} P_{\mu}\left(M_{k}\right)=P_{\mu}(M) .
$$

4) If $M$ is a Borel set in $\mathbb{R}^{n}$, then

$$
P_{\mu}(M) \leq \mu^{+}(M)
$$

We mention that the theory of sets with finite $\mu$-perimeter is imbedded in the framework of $\mathrm{BV}$-functions space, $B V\left(\varphi, \mathbb{R}^{n}\right)$, defined as the set of all functions $u \in L^{1}\left(\varphi, \mathbb{R}^{n}\right)$ such that

$$
\|D u\|_{B V}:=\sup \left\{\int_{\mathbb{R}^{n}} u(x) \operatorname{div}(\varphi(x) h(x)) d x, h \in C_{0}^{1}\left(\mathbb{R}^{n}, \mathbb{R}^{n}\right),|h| \leq 1\right\}<+\infty .
$$

Notice that if $M$ has finite $\mu$-perimeter then the characteristic function $\chi_{M}$ of $M$ belongs to $B V\left(\varphi, \mathbb{R}^{n}\right)$ and moreover $\left\|D \chi_{M}\right\|_{B V}=P_{\mu}(M)$. Furthermore, if $u \in$ $W^{1,1}\left(\varphi, \mathbb{R}^{n}\right)$ then $\|D u\|_{B V}=\|\nabla u\|_{1}$.

Finally we recall some well-known derivation formulas (cf. [1], [18] and [11]).

We set $\mathbb{R}_{0}^{+}=[0,+\infty)$ and we denote a point $x \in \mathbb{R}^{n}$ by $x=\left(x_{1}, x^{\prime}\right)$ where $x_{1} \in \mathbb{R}$ and $x^{\prime} \in \mathbb{R}^{n-1}(n \geq 2)$.

Let $u \in \mathcal{F}^{n}$, and let $m_{u}$ denote its $\mu$-distribution function w.r.t. the variable $x_{1}$, that is, ${ }^{1}$

$$
m_{u}\left(t, x^{\prime}\right):=\mu_{1}\left(\left\{u\left(\cdot, x^{\prime}\right)>t\right\}\right), \quad t \in \mathbb{R}, x^{\prime} \in \mathbb{R}^{n-1} .
$$

We also set

$$
u_{-}:=\operatorname{ess} \inf u
$$

and

$$
V_{u}:=\left\{\left(t, x^{\prime}\right): t>u_{-}, x^{\prime} \in \mathbb{R}^{n-1}\right\} .
$$

Let $u \in W^{1, p}\left(\varphi, \mathbb{R}^{n}\right)$ for some $p \in[1,+\infty)$. We define

$$
D_{u}\left(x^{\prime}\right):=\left\{x_{1} \in \mathbb{R}: u_{x_{1}}\left(x_{1}, x^{\prime}\right)=0\right\}, \quad x^{\prime} \in \mathbb{R}^{n-1},
$$

and the superlevel sets

$$
E_{u}\left(t, x^{\prime}\right):=\left\{u\left(x_{1}, x^{\prime}\right)>t\right\} \equiv\left\{u\left(\cdot, x^{\prime}\right)>t\right\}, \quad\left(t, x^{\prime}\right) \in V_{u} .
$$

Notice that, since $u\left(\cdot, x^{\prime}\right) \in W^{1, p}(\varphi, \mathbb{R})$ for a.e. $x^{\prime} \in \mathbb{R}^{n-1}$, the Sobolev imbedding theorem tells us that $u\left(\cdot, x^{\prime}\right)$ is continuous for a.e. $x^{\prime} \in \mathbb{R}^{n-1}$. Hence $E_{u}\left(t, x^{\prime}\right)$ is open and $\partial E_{u}\left(t, x^{\prime}\right)$ is countable for a.e. $\left(t, x^{\prime}\right) \in V_{u}$.

\footnotetext{
${ }^{1}$ Here and in the following we will write $\left\{u\left(\cdot, x^{\prime}\right)>t\right\}$ for $\left\{x_{1}: u\left(x_{1}, x^{\prime}\right)>t\right\}$.
} 
Let us consider $g \in L^{1}\left(\mathbb{R}^{n}\right)$. Defining

$$
F\left(t, x^{\prime}\right):=\int_{E_{u}\left(t, x^{\prime}\right)} g\left(x_{1}, x^{\prime}\right) d x_{1}, \quad\left(t, x^{\prime}\right) \in \mathbb{R} \times \mathbb{R}^{n-1},
$$

we then have that $F\left(\cdot, x^{\prime}\right) \in B V(\mathbb{R})$ for a.e. $x^{\prime} \in \mathbb{R}^{n-1}$, and the Fleming-Rishel formula (see, for instance, [17]) tells us that

$$
\frac{\partial}{\partial t} F\left(t, x^{\prime}\right)=\int_{\partial E_{u}\left(t, x^{\prime}\right)} \frac{g\left(\cdot, x^{\prime}\right)}{\left|u_{x_{1}}\left(\cdot, x^{\prime}\right)\right|} d \mathcal{H}_{0} \quad \text { for a.e. }\left(t, x^{\prime}\right) \in V_{u} .
$$

Moreover, we have by the co-area formula (see, e.g., [17])

$$
\int_{\mathbb{R}} F\left(t, x^{\prime}\right) d t=\int_{\mathbb{R} \backslash D_{u}\left(x^{\prime}\right)} g\left(x_{1}, x^{\prime}\right) d x_{1}=\int_{u_{-}\left(x^{\prime}\right)}^{u_{+}\left(x^{\prime}\right)} \int_{\partial E_{u}\left(t, x^{\prime}\right)} \frac{g\left(\cdot, x^{\prime}\right)}{\left|u_{x_{1}}\left(\cdot, x^{\prime}\right)\right|} d \mathcal{H}_{0} d t
$$

for a.e. $x^{\prime} \in \mathbb{R}^{n-1}$.

Now let us assume that $u$ is a smooth function and satisfies

$$
\mathcal{L}^{1}\left(D_{u}\left(x^{\prime}\right)\right)=0 \quad \text { for a.e. } x^{\prime} \in \mathbb{R}^{n-1} .
$$

Here $\mathcal{L}^{1}$ denotes the one-dimensional Lebesgue measure. Then the following derivation formula holds true (cf [1]; see also [18] and [11]).

Lemma 2.1. If $g \in C^{1}$, then we have

$$
\begin{aligned}
\frac{\partial}{\partial x_{i}} & \int_{E_{u}\left(t, x^{\prime}\right)} g\left(x_{1}, x^{\prime}\right) d x_{1} \\
& =\int_{E_{u}\left(t, x^{\prime}\right)} \frac{\partial g}{\partial x_{i}}\left(x_{1}, x^{\prime}\right) d x_{1}+\int_{\partial E_{u}\left(t, x^{\prime}\right)} g\left(\cdot, x^{\prime}\right) \frac{u_{x_{i}}\left(\cdot, x^{\prime}\right)}{\left|u_{x_{1}}\left(\cdot, x^{\prime}\right)\right|} d \mathcal{H}_{0} .
\end{aligned}
$$

\section{Measures on the real line}

Let $\psi$ a positive, even and continuous function on $\mathbb{R}$, such that

$$
\int_{\mathbb{R}} \psi(x) d x=+\infty
$$

We then define a measure $\mu_{1}$ on $\mathbb{R}$ by

$$
d \mu_{1}=\psi(x) d x
$$

The primitive of $\psi$,

$$
\Psi(x):=\int_{0}^{x} \psi(t) d t, \quad x \in \mathbb{R},
$$

is strictly increasing and odd. 
We introduce a continuous, positive function $J$ by

$$
J(y)=\psi\left(\Psi^{-1}(y)\right), \quad y \in \mathbb{R},
$$

where $\Psi^{-1}: \mathbb{R} \rightarrow \mathbb{R}$ is the inverse of $\Psi$. Notice that $J$ is even, and (3.1) implies that

$$
\Psi^{-1}(y)=\int_{0}^{y} \frac{d t}{J(t)}, \quad y \in \mathbb{R} .
$$

If $M \in \mathcal{M}^{1}$, and if $\mu_{1}(M)<+\infty$, then there exists a unique number $c \geq 0$ such that

$$
\mu_{1}((-c, c))=\mu_{1}(M)
$$

and we set

$$
M^{*}=(-c, c)
$$

If $M \in \mathcal{M}^{1}$, and if $\mu_{1}(M)=+\infty$ then we set $M^{*}=\mathbb{R}$. We call the set $M^{*}$ the $\mu_{1}$-symmetrization of $M$.

Remark 3.1. By the above definition, $M^{*}$ is some centered interval $(-c, c),(c \in$ $\left.\mathbb{R}_{0}^{+} \cup\{+\infty\}\right)$. Notice that since we deal with measurable sets we usually do not distinguish between two sets $M, N$ which are equivalent, that is, which satisfy $\mu_{1}(M \Delta N)=0$. If $M$ is closed, we take $M^{*}$ to be the closed interval $[-c, c]$.

Remark 3.2. Obviously, by definition

$$
\mu_{1}(M)=\mu_{1}\left(M^{*}\right) \quad \forall M \in \mathcal{M}^{1} .
$$

It is also easy to confirm the following monotonicity properties, $\left(M, N \in \mathcal{M}^{1}\right)$ :

$$
\begin{aligned}
& M \subset N \Longrightarrow M^{*} \subset N^{*}, \\
& M^{*} \cup N^{*} \subset(M \cup N)^{*}, \quad M^{*} \cap N^{*} \supset(M \cap N)^{*}, \\
& \mu_{1}(M \backslash N) \geq \mu_{1}\left(M^{*} \backslash N^{*}\right), \quad \mu_{1}(M \Delta N) \geq \mu_{1}\left(M^{*} \Delta N^{*}\right) .
\end{aligned}
$$

We now ask for a condition on the measure $\mu_{1}$ such that the $\mu_{1}$-rearrangement decreases the perimeter, that is we ask for a condition such that the following isoperimetric inequality holds:

$$
P_{\mu_{1}}(M) \geq P_{\mu_{1}}\left(M^{*}\right) \equiv J\left(\mu_{1}(M)\right), \quad \forall M \in \mathcal{M}^{1} .
$$

Such a condition is given by Theorem 3.3 below. This theorem can be subsumed by more general results of Section 3 of [28]; here we give the proof in our specific case.

Theorem 3.3. Inequality (3.9) holds if and only if $J$ is convex. Furthermore, let us assume that equality holds in (3.9); if $J$ is convex, then $M$ is equivalent to an interval, while if $J$ is strictly convex, then $M=M^{*}$. 
Remark 3.4. $J$ is convex if and only if $\log \psi$ is convex (or equivalently, if $\psi$ is log-convex). A typical case is

$$
\psi(x)=e^{c|x|^{2}},
$$

where $c \in \mathbb{R}_{0}^{+}$. Obviously if $c>0$ then $J$ is strictly convex.

Remark 3.5. The isoperimetric inequality (3.9) and property (2.11) imply an isoperimetric inequality for the Minkowsky $\mu_{1}$-content, i.e.,

$$
\mu_{1}^{+}(M) \geq \mu_{1}^{+}\left(M^{*}\right) \quad \text { for every Borel set } M .
$$

Moreover it is easy to see that this also implies

$$
\mu_{1}\left(M_{r}\right) \geq \mu_{1}\left(\left(M^{*}\right)_{r}\right) \quad \forall r>0,
$$

which is equivalent to

$$
\left(M_{r}\right)^{*} \supset\left(M^{*}\right)_{r} \quad \forall r>0 .
$$

Proof of Theorem 3.3. First let us assume that $\mu_{1}$ satisfies (3.9), and let $I$ be any finite interval $(a, b),(a<b)$. Setting $\alpha=\Psi(a), \beta=\Psi(b)$, we have that $I^{*}=(-c, c)$, where $\Psi(c)=(\beta-\alpha) / 2$. Then (3.9) reads

$$
J(\alpha)+J(\beta) \geq 2 J\left(\frac{\beta-\alpha}{2}\right) .
$$

Since $J(\alpha)=J(-\alpha)$, this implies that

$$
J(s)+J(t) \geq 2 J\left(\frac{s+t}{2}\right) \quad \forall s, t \in \mathbb{R},
$$

which means that $J$ is convex.

Now let us assume that $J$ is convex, and let $M$ be a smooth set. Then $M=$ $\cup_{i=1}^{m}\left(a_{i}, b_{i}\right)$ where $m \in \mathbb{N}, a_{i}<b_{i}$, and the intervals $\left[a_{i}, b_{i}\right]$ are mutually disjoint, $i=1,2, \ldots, m$. Setting $\alpha_{i}=\Psi\left(a_{i}\right)$ and $\beta_{i}=\Psi\left(b_{i}\right), i=1, \ldots, m$, we find, using that $J$ is convex and even,

$$
\begin{aligned}
P_{\mu_{1}}(M) & =\sum_{i=1}^{m}\left(J\left(\alpha_{i}\right)+J\left(\beta_{i}\right)\right) \geq \sum_{i=1}^{m} 2 J\left(\frac{\beta_{i}-\alpha_{i}}{2}\right) \\
& \geq 2 m J\left(\sum_{i=1}^{m} \frac{\beta_{i}-\alpha_{i}}{2 m}\right) \geq 2 J\left(\sum_{i=1}^{m} \frac{\beta_{i}-\alpha_{i}}{2}\right)=\mu_{1}^{+}\left(M^{*}\right) .
\end{aligned}
$$

By property (2.10) this also implies (3.9) for sets $M \in \mathcal{M}^{1}$, proving the first statement of the theorem.

Now we assume that equality holds in (3.9) and that $J$ is convex. Without loss of generality we may assume that also $\mu_{1}(M)>0$. For $r>0$, let $\theta_{r}(M, x):=$ $\mu_{1}\left(M \cap B_{r}(x)\right)\left[\mu_{1}\left(B_{r}(x)\right)\right]^{-1}$ and define the upper density of the set $M$ at $x$ by $\theta(M, x):=\lim \sup _{r \searrow 0} \theta_{r}(M, x)$. Suppose that $M^{\prime}$ is the set of upper density points of $M$, i.e. $M^{\prime}=\{x \in \mathbb{R}: \theta(M, x)=1\}$. Since $\mu_{1}\left(M \Delta M^{\prime}\right)=0$, it is sufficient to study $M^{\prime}$ instead of $M$. We first claim that $M^{\prime}$ is convex. 
Assume that $M^{\prime}$ is not convex. Then there are points $x_{i} \in \mathbb{R}, i=1,2,3$, $x_{1}<x_{3}<x_{2}$, such that $\theta\left(M, x_{1}\right)=\theta\left(M, x_{2}\right)=1$ and $\theta\left(M, x_{3}\right)=0$. Then for $r<$ $(1 / 4) \min \left\{\left(x_{3}-x_{1}\right) ;\left(x_{2}-x_{3}\right)\right\}$, and small enough, we have that $\theta_{r}\left(M, x_{i}\right) \geq 7 / 8$, $i=1,2$, and $\theta_{r}\left(M, x_{3}\right) \leq 1 / 8$. Let $\left\{M_{k}\right\}$ be a sequence of smooth sets such that $\mu_{1}\left(M_{k} \Delta M\right) \rightarrow 0$ and $P_{\mu_{1}}\left(M_{k}\right) \rightarrow P_{\mu_{1}}(M)$, as $k \rightarrow \infty$. For $k$ large enough (say $\left.k \geq k_{0}\right)$ we still have $\theta_{r}\left(M_{k}, x_{i}\right) \geq 3 / 4, i=1,2$, and $\theta_{r}\left(M_{k}, x_{3}\right) \leq 1 / 4$. This implies that the sets $B_{r}\left(x_{i}\right) \cap M_{k}, i=1,2$, and $B_{r}\left(x_{3}\right) \backslash M_{k}$ are nonempty for these $k$. In other words, if $k \geq k_{0}$, then there is a nonempty interval $I_{k}=\left(y_{k}^{1}, y_{k}^{2}\right)$ such that $I_{k} \subset\left(x_{1}-r, x_{2}+r\right), I_{k} \cap M_{k}=\emptyset$ and $y_{k}^{1}, y_{k}^{2} \in \partial M_{k}$. Setting $N_{k}:=I_{k} \cup M_{k}$ we then have in view of the isoperimetric property (3.9),

$$
\begin{aligned}
P_{\mu_{1}}\left(M_{k}\right)-J\left(\mu_{1}\left(M_{k}\right)\right) & \geq P_{\mu_{1}}\left(N_{k}\right)-J\left(\mu_{1}\left(N_{k}\right)\right)+\psi\left(y_{k}^{1}\right)+\psi\left(y_{k}^{2}\right) \\
& \geq \psi\left(y_{k}^{1}\right)+\psi\left(y_{k}^{2}\right) \geq \delta, \quad \forall k \geq k_{0},
\end{aligned}
$$

for some $\delta>0$ independent on $k$. Passing to the limit for $k \rightarrow \infty$, this also implies $P_{\mu_{1}}(M)-J\left(\mu_{1}(M)\right) \geq \delta>0$, which is a contradiction. Hence $M^{\prime}$ is convex.

Now we assume that equality holds in (3.9) and that $J$ is strictly convex. Hence $M^{\prime}=(a, b)$, where $a, b \in \mathbb{R}, a<b$. Setting $\alpha=\Psi(a)$ and $\beta=\Psi(b)$, we have that $M^{*}=(-c, c)$, where $\Psi(c)=(\beta-\alpha) / 2$, so that

$$
J(\alpha)+J(\beta)=2 J\left(\frac{\beta-\alpha}{2}\right) .
$$

The strict convexity of $J$ then implies that $|\alpha|=|\beta|$, that is $b=-a$. The theorem is proved.

\section{Product measures on $\mathbb{R}^{n}$}

In this section we prove isoperimetric inequalities with respect to product measures on $\mathbb{R}^{n}$ and we apply them to obtain integral inequalities in Sobolev spaces.

We deal with a product measure $\mu$ on $\mathbb{R}^{n}$ defined by

$$
d \mu=\psi\left(x_{1}\right) \rho\left(x^{\prime}\right) d x
$$

where $x=\left(x_{1}, x_{2}, \ldots, x_{n}\right)=\left(x_{1}, x^{\prime}\right)$ is a point in $\mathbb{R}^{n},(n \geq 2), \psi$ is a function as in the previous section and $\rho$ is a positive, continuous function on $\mathbb{R}^{n-1}$.

\subsection{Isoperimetric inequalities}

If $M \subset \mathbb{R}^{n}$ we introduce $x^{\prime}$ - slices, $x^{\prime} \in \mathbb{R}^{n-1}$, by

$$
M\left(x^{\prime}\right)=\left\{x_{1}:\left(x_{1}, x^{\prime}\right) \in M\right\} .
$$

Notice that if $M \in \mathcal{M}^{n}$, and if $\mu(M)<\infty$ then $\mu_{1}\left(M\left(x^{\prime}\right)\right)<\infty$ for almost every $x^{\prime} \in \mathbb{R}^{n-1}$. For $M \in \mathcal{M}^{n}$, we define its Steiner $\mu$-symmetrization $M^{*}$ by

$$
M^{*}:=\left\{x=\left(x_{1}, x^{\prime}\right): x_{1} \in\left(M\left(x^{\prime}\right)\right)^{*}, x^{\prime} \in \mathbb{R}^{n-1}\right\} .
$$

Then

$$
\mu(M)=\mu\left(M^{*}\right)
$$


and it is easy to see that the monotonicity properties (3.6)-(3.8) carry over to Steiner $\mu$-symmetrization.

As in the one-dimensional case, the above definitions will be interpreted pointwise for open and closed sets.

If $M$ is open (resp., closed) then the function $\varphi\left(x^{\prime}\right):=\mu\left(M\left(x^{\prime}\right)\right),\left(x^{\prime} \in \mathbb{R}^{n-1}\right)$, is lower (resp., upper) semicontinuous. Since the sets $\left(M\left(x^{\prime}\right)\right)^{*},\left(x^{\prime} \in \mathbb{R}^{n-1}\right)$, are open (resp., closed) it then follows that $M^{*}$ is open (resp., closed), too.

Steiner-like rearrangements are characterized by the fact that the isoperimetric property (3.11) on slices carries over to sets on $\mathbb{R}^{n}$ (see [10]). By adapting the proof of Theorem 3.3 in [6], we can prove the following result

Lemma 4.1. The property (3.11) holds for Borel sets $M$ on $\mathbb{R}^{n}$.

By the definition of $\mu^{+}$and $P_{\mu}$, and by property $(2.10)$ we then also have

Corollary 4.2. The following inequalities hold:

$$
\begin{aligned}
& \mu^{+}(M) \geq \mu^{+}\left(M^{*}\right) \quad \text { for every Borel set } M, \text { and } \\
& P_{\mu}(M) \geq P_{\mu}\left(M^{*}\right) \quad \text { for every } M \in \mathcal{M}^{n} .
\end{aligned}
$$

The next property follows easily from Lemma 4.1.

Corollary 4.3. Let $M$ and $N$ be open sets in $\mathbb{R}^{n}$ with $M \subset N$. Then

$$
\operatorname{dist}\{M ; \partial N\} \leq \operatorname{dist}\left\{M^{*} ; \partial N^{*}\right\} .
$$

Finally we analyze the equality case in (4.5). The following result holds:

Theorem 4.4. Assume that equality holds in (4.5) for some $M \in \mathcal{M}^{n}$. Then $M\left(x^{\prime}\right)$ is either empty or equivalent to an interval for almost every $x^{\prime} \in \mathbb{R}^{n-1}$. Moreover, if $J$ is strictly convex, then $M=M^{*}$.

The proof of Theorem 4.4 depends on a precise estimate for the deficit of the perimeter under Steiner symmetrization for polyhedra. This approach is wellknown in the case of the uniform Lebesgue measure $\varphi \equiv 1$ (see chapter 14 of [10]). Let us first introduce some notation. Let $\nabla^{\prime}$ denote the vector of derivatives $\left(\partial / \partial x_{2}, \ldots, \partial / \partial x_{n}\right)$. If $x^{\prime} \in \mathbb{R}^{n-1}$ then let $l_{x^{\prime}}$ denote the line $\left\{\left(t, x^{\prime}\right): t \in \mathbb{R}\right\}$. Let $\mathcal{P}$ denote the set of polyhedra $\Pi$ in $\mathbb{R}^{n}$ such that $l_{x^{\prime}} \cap \Pi$ is either empty or consists of a finite number of points for every $x^{\prime} \in \mathbb{R}^{n-1}$. The map $p: \partial \Pi \rightarrow \mathbb{R}^{n-1}$ will be called a projection. If $\Pi \in \mathcal{P}$ then $\mathbb{R}^{n-1}$ is splitted into a finite numbers of domains $Q$ such that the part of $\partial \Pi$ which is projected into $Q$ consists of a finite number $2 m$ of components $\Gamma_{j}$ whose projections onto $Q$ are one-to-one. (The number $m$ depends on $Q$, and those for which $m=0$ will not be considered further on.)

Each $\Gamma_{j}$ permits an (affine) representation $x_{1}=z_{j}\left(x^{\prime}\right), x^{\prime} \in Q$. Then it follows that

$$
P_{\mu}(\Pi)=\sum * \int_{Q} \sum_{j=1}^{2 m} \sqrt{1+\left|\nabla^{\prime} z_{j}\right|^{2}} \psi\left(z_{j}\right) \rho\left(x^{\prime}\right) d x^{\prime}
$$


where the sum $\sum^{*}$ is taken over all the $Q$ for which $m \geq 1$. After the Steiner symmetrization, the area of the boundary will be

$$
P_{\mu}\left(\Pi^{*}\right)=\sum * \int_{Q} 2 \sum_{j=1}^{2 m} \sqrt{1+\left|\nabla^{\prime} z\right|^{2}} \psi(z) \rho\left(x^{\prime}\right) d x^{\prime}
$$

where the function $z: Q \rightarrow \mathbb{R}_{0}^{+}$is given by

$$
\sum_{j=1}^{2 m}(-1)^{j} \Psi\left(z_{j}\right)=2 \Psi(z)
$$

Lemma 4.5. Let $\Pi \in \mathcal{P}$. Then, with the above notations,

$$
P_{\mu}(\Pi)-P_{\mu}\left(\Pi^{*}\right)
$$

$$
\geq\left(P_{\mu}\left(\Pi^{*}\right)\right)^{-1} \sum^{*} \int_{Q}\left(\psi(z)\left|\sum_{j=1}^{2 m} \psi\left(z_{j}\right)-2 \psi(z)\right|\right)^{1 / 2} \rho\left(x^{\prime}\right) d x^{\prime} .
$$

Proof. For convenience, we set $y_{j}:=\Psi\left(z_{j}\right), j=1, \ldots 2 m, y=\Psi(z)$, and $J:=$ $\psi\left(\Psi^{-1}\right)$. Recall that $J$ is convex by our assumptions. Then we find

$$
\begin{aligned}
\sum_{j=1}^{2 m} \sqrt{1+\left|\nabla^{\prime} z_{j}\right|^{2}} \psi\left(z_{j}\right)-2 \sqrt{1+\left|\nabla^{\prime} z\right|^{2}} \psi(z) & \\
& =\sum_{j=1}^{2 m} \sqrt{J\left(y_{j}\right)^{2}+\left|\nabla^{\prime} y_{j}\right|^{2}}-2 \sqrt{J(y)^{2}+\left|\nabla^{\prime} y\right|^{2}} \\
& \geq \frac{J(y)}{\sqrt{J(y)^{2}+\left|\nabla^{\prime} y\right|^{2}}}\left(\sum_{j=1}^{2 m} J\left(y_{j}\right)-2 J(y)\right)=\frac{\sum_{j=1}^{2 m} \psi\left(z_{j}\right)-2 \psi(z)}{\sqrt{1+\left|\nabla^{\prime} z\right|^{2}}} .
\end{aligned}
$$

Integrating (4.11) and applying the Cauchy-Schwarz inequality we have (4.10).

Proof of Theorem 4.4. For $x \in \mathbb{R}^{n}$ and $r>0$ let

$$
\theta_{r}(M, x):=\mu\left(M \cap B_{r}(x)\right)\left[\mu\left(B_{r}(x)\right)\right]^{-1},
$$

and define $\theta\left(M, x^{\prime}\right)$ and the set $M^{\prime}$ of upper density points of $M$ as in the proof of Theorem 3.3. As before, we may restrict ourselves to the set $M^{\prime}$ instead of $M$.

Choose a sequence of polyhedra $\left\{\Pi_{k}\right\}$ such that $\mu\left(\Pi_{k} \Delta M\right) \rightarrow 0$ and $P_{\mu}\left(\Pi_{k}\right) \rightarrow$ $P_{\mu}(M)$ as $k \rightarrow \infty$. Without loss of generality, we may also assume that $\Pi_{k} \in \mathcal{P}$, $k=1,2, \ldots$ Since $\mu\left(\Pi_{k}^{*} \Delta M^{*}\right) \rightarrow 0$ as $k \rightarrow \infty$, we have by the lower semicontinuity of the perimeter,

$$
\lim _{k \rightarrow \infty}\left(P_{\mu}\left(\Pi_{k}\right)-P_{\mu}\left(\Pi_{k}^{*}\right)\right)=0 .
$$

Set

$$
R_{k}:=\left\{x^{\prime} \in \mathbb{R}^{n-1}: l_{x^{\prime}} \cap \Pi_{k} \text { has at least two components }\right\},
$$

and introduce a measure $\nu$ on $\mathbb{R}^{n-1}$ defined by

$$
d \nu=\rho\left(x^{\prime}\right) d x^{\prime} .
$$


Since the function $\psi$ is bounded away from 0, Lemma 4.10 together with (4.12) tells us that

$$
\lim _{k \rightarrow \infty} \nu\left(R_{k}\right)=0
$$

We claim that $M\left(x^{\prime}\right)$ is convex for almost every $x^{\prime} \in \mathbb{R}^{n-1}$.

Assume that this is not the case. Then there are points $x^{i}=\left(z_{i}, x_{0}^{\prime}\right), i=1,2,3$, with $x_{0}^{\prime} \in \mathbb{R}^{n-1}$, and $z_{1}<z_{3}<z_{2}$, such that $\theta\left(M, x^{1}\right)=\theta\left(M, x^{2}\right)=1$, and $\theta\left(M, x^{3}\right)=0$. Let $\varepsilon$ be positive and small (the exact choice of $\varepsilon$ will be specified later). Choose $r(=r(\varepsilon))>0$, and small enough such that $\theta_{r}\left(M, x^{i}\right) \geq 1-\varepsilon$, $i=1,2$, and $\theta_{r}\left(M, x^{3}\right) \leq \varepsilon$. For $k$ large enough - say $k \geq k_{\varepsilon}-$ we then still have $\theta_{r}\left(\Pi_{k}, x^{i}\right) \geq 1-2 \varepsilon, i=1,2$, and $\theta_{r}\left(\Pi_{k}, x^{3}\right) \leq 2 \varepsilon$. Let

$H_{k}:=\left\{x=\left(x_{1}, x^{\prime}\right) \in B_{r}\left(x^{3}\right): \nu\left(l_{x^{\prime}} \cap \Pi_{k} \cap B_{r}\left(x^{1}\right)\right)>0, \nu\left(l_{x^{\prime}} \cap \Pi_{k} \cap B_{r}\left(x^{2}\right)\right)>0\right\}$.

By choosing $\varepsilon$ small enough we can achieve that $\mu\left(H_{k}\right)>(1 / 2) \mu\left(B_{r}\left(x^{3}\right)\right)$, and in view of $\theta_{r}\left(\Pi_{k}, x^{3}\right) \leq 2 \varepsilon$, also that $\mu\left(H_{k} \backslash \Pi_{k}\right)>(1 / 4) \mu\left(B_{r}\left(x^{3}\right)\right)$. Hence there is a number $c_{0}>0$ which depends only on $\varepsilon$, but not on $k$, such that $\nu\left(R_{k}\right) \geq c_{0}$. This contradicts (4.13), and proves the claim.

Hence there is a nullset $N \subset \mathbb{R}^{n}$, a measurable set $G \subset \mathbb{R}^{n-1}$, and measurable functions $z_{i}, i=1,2$, such that

$$
M=N \cup\left\{\left(x_{1}, x^{\prime}\right): z_{1}\left(x^{\prime}\right)<x_{1}<z_{2}\left(x^{\prime}\right), x^{\prime} \in G\right\} .
$$

Using Lemma 4.10 and the limit property (4.12) we have

$$
0=P_{\mu}(M)-P_{\mu}\left(M^{*}\right)
$$

$$
\geq\left(P_{\mu}(M)\right)^{-1} \int_{G}\left(\psi(z)\left|\sum_{j=1}^{2} \psi\left(z_{j}\right)-2 \psi(z)\right|\right)^{1 / 2} \rho\left(x^{\prime}\right) d x^{\prime},
$$

where $z$ is given by $2 \Psi(z)=\Psi\left(z_{2}\right)-\Psi\left(z_{1}\right)$. Using the strict convexity of $J$ this implies that $z_{2}=-z_{1}=z$ on $G$ and the theorem is proved.

\subsection{Steiner $\mu$-symmetrization of functions}

If $u \in \mathcal{F}^{n}$ we define its Steiner $\mu$-symmetrization (w.r.t. $x_{1}$ ) $u^{*}$ by

$$
u^{*}(x):=\sup \left\{t \in \mathbb{R}: x \in\{u>t\}^{*}\right\}, \quad x \in \mathbb{R}^{n} .
$$

By its definition, the function $u^{*}$ is nonincreasing and right-continuous w.r.t. the variable $x_{1}$. Moreover $u$ and $u^{*}$ are equimeasurable functions, that is

$$
\{u>t\}^{*}=\left\{u^{*}>t\right\} \quad \text { and } \quad\{u \geq t\}^{*}=\left\{u^{*} \geq t\right\} \quad \forall t>\inf u,
$$

which implies that, for all $t>\inf u$ and for a.e. $x^{\prime} \in \mathbb{R}^{n-1}$,

$$
\begin{aligned}
\mu(\{u>t\}) & =\mu\left(\left\{u^{*}>t\right\}\right), \mu(\{u \geq t\})=\mu\left(\left\{u^{*} \geq t\right\}\right), \text { and } \\
\mu\left(\left\{u\left(\cdot, x^{\prime}\right)>t\right\}\right) & =\mu\left(\left\{u^{*}\left(\cdot, x^{\prime}\right)>t\right\}\right), \mu\left(\left\{u\left(\cdot, x^{\prime}\right) \geq t\right\}\right)=\mu\left(\left\{u^{*}\left(\cdot, x^{\prime}\right) \geq t\right\}\right) .
\end{aligned}
$$

Furthermore, the monotonicity (3.6) implies

$$
u, v \in \mathcal{F}^{n}, u \leq v \Longrightarrow u^{*} \leq v^{*} .
$$


Remark 4.6. We will generally not distinguish between $u$ and its equivalence class given by all measurable functions which differ from $u$ on a nullset. But if $u$ is continuous, then the sets $\{u>t\},(t \in \mathbb{R})$ are open, and the above definition of $u^{*}$ has to be understood in pointwise sense. Furthermore it is easy to see that the sets $\left\{u^{*}>t\right\},\left(\right.$ respectively $\left.\left\{u^{*} \geq t\right\}\right)$ are open (respectively closed), $(t \in \mathbb{R})$, so that $u^{*}$ is continuous too.

Remark 4.7. An equivalent definition of $u^{*}$ can be given by using the $\mu$-distribution function of $u$ (w.r.t. $x_{1}$ ), $m_{u}$, defined by

$$
m_{u}\left(x^{\prime}, t\right):=\mu_{1}\left(\left\{u\left(\cdot, x^{\prime}\right)>t\right\}\right), \quad x^{\prime} \in \mathbb{R}^{n-1}, t>\inf u .
$$

The function $m_{u}$ is nonnegative, nonincreasing, and right-continuous w.r.t. the variable $t$, and

$$
u^{*}\left(x_{1}, x^{\prime}\right)=\sup \left\{t \in \mathbb{R}: m_{u}\left(x^{\prime}, t\right)>\mu_{1}\left(\left(-x_{1}, x_{1}\right)\right)\right\}, \quad x=\left(x_{1}, x^{\prime}\right) \in \mathbb{R}^{n} .
$$

Proceeding analogously as we did in [6] for a certain class of Steiner-like rearrangements w.r.t. a finite measure, we can prove the following properties:

Theorem 4.8. 1) If $u \in L_{+}^{1}\left(\mu, \mathbb{R}^{N}\right)$, then

$$
\int_{\mathbb{R}^{n}} u d \mu=\int_{\mathbb{R}^{n}} u^{*} d \mu .
$$

2) If $u \in \mathcal{F}^{n}$ and if $\varphi: \mathbb{R} \rightarrow \mathbb{R}$ is a nondecreasing function, then

$$
\varphi\left(u^{*}\right)=(\varphi(u))^{*} .
$$

3) (Cavalieri's principle). If $f: \mathbb{R} \rightarrow \mathbb{R}$ is continuous or nondecreasing, $u \in \mathcal{F}^{n}$ and if $f(u) \in L^{1}\left(\mu, \mathbb{R}^{n}\right)$, then $f\left(u^{*}\right) \in L^{1}\left(\mu, \mathbb{R}^{n}\right)$ and

$$
\int_{\mathbb{R}^{n}} f(u) d \mu=\int_{\mathbb{R}^{n}} f\left(u^{*}\right) d \mu .
$$

4) Let $F \in C\left(\left(\mathbb{R}_{0}^{+}\right)^{2}\right), F(0,0)=0$, and

$$
F(A, B)-F(a, B)-F(A, b)+F(a, b) \geq 0
$$

for all $a, b, A, B \in \mathbb{R}_{0}^{+}$with $a \leq A, b \leq B$.

Furthermore, let $u, v \in \mathcal{F}_{+}^{n}$ be such that $F(u, 0), F(0, v), F(u, v) \in L^{1}\left(\mu, R^{n}\right)$. Then

$$
\int_{\mathbb{R}^{n}} F(u, v) d \mu \leq \int_{\mathbb{R}^{n}} F\left(u^{*}, v^{*}\right) d \mu .
$$

5) (Nonexpansivity of the rearrangement). Let $G \in C\left(\mathbb{R}_{0}^{+}\right)$be continuous, nondecreasing and convex with $G(0)=0$, and let $u, v \in \mathcal{F}_{+}^{n}$ be such that $G(|u|)$, $G(|v|), G(|u-v|) \in L^{1}\left(\mu, \mathbb{R}^{n}\right)$. Then

$$
\int_{\mathbb{R}^{n}} G\left(\left|u^{*}-v^{*}\right|\right) d \mu \leq \int_{\mathbb{R}^{n}} G(|u-v|) d \mu .
$$


6) Let $u, v \in L_{+}^{2}\left(\mu, \mathbb{R}^{n}\right)$. Then

$$
\int_{\mathbb{R}^{n}} u v d \mu \leq \int_{\mathbb{R}^{n}} u^{*} v^{*} d \mu .
$$

7) Let $u, v \in L^{\infty}\left(\mathbb{R}^{n}\right) \cap \mathcal{F}^{n}$. Then

$$
\left\|u^{*}-v^{*}\right\|_{\infty} \leq\|u-v\|_{\infty} .
$$

Remark 4.9. If $F \in C^{2}$ then (4.23) is equivalent to

$$
\frac{\partial^{2} F(\sigma, \tau)}{\partial \sigma \partial \tau} \geq 0, \quad \forall \sigma, \tau \in \mathbb{R}_{0}^{+} .
$$

Proof. The proofs of the properties 1)-3), and of 5)-7), mimic the proofs of analogous properties in [6].

4) We proceed similarly as in [12]. In view of (4.23), there exists a nonnegative measure, denoted by $d F_{\sigma \tau}$, such that

$$
\begin{aligned}
F(s, t)-F(s, 0)-F(0, t) & =\int_{0}^{t} \int_{0}^{s} d F_{\sigma \tau} \\
& =\iint_{\left(\mathbb{R}_{0}^{+}\right)^{2}} \chi(0, s)(\sigma) \chi(0, t)(\tau) d F_{\sigma \tau} \quad \forall s, t \in \mathbb{R}_{0}^{+} .
\end{aligned}
$$

Notice that in the case $F \in C^{2}$ we have

$$
d F_{\sigma \tau}=\frac{\partial^{2} F(\sigma, \tau)}{\partial \sigma \partial \tau} d \sigma d \tau,
$$

which is obviously nonnegative by $(4.23)$.

Choosing $s=u(x)$ and $t=v(x)$ in (4.29) and then integrating we find

$$
\begin{aligned}
\int_{\mathbb{R}^{n}} F(u, v) d \mu= & \int_{\mathbb{R}^{n}} F(u, 0) d \mu+\int_{\mathbb{R}^{n}} F(0, v) d \mu \\
& +\int_{\left(\mathbb{R}_{0}^{+}\right)^{2}} \mu(\{u>\sigma\} \cap\{v>\tau\}) d F_{\sigma \tau} .
\end{aligned}
$$

An analogous expression for $\int_{\mathbb{R}^{n}} F\left(u^{*}, v^{*}\right) d \mu$ holds. Since from (3.7), we have

$$
\mu(\{u>\sigma\} \cap\{v>\tau\}) \leq \mu\left(\left\{u^{*}>\sigma\right\} \cap\left\{v^{*}>\tau\right\}\right) \quad \forall \sigma, \tau>0 .
$$

Then (4.24) follows from (4.30) and (4.31).

Remark 4.10. Let $M=\mathbb{R} \times M^{\prime}$, where $M \in \mathcal{M}^{n-1}$, and let $u, v \in \mathcal{F}^{n}$. Then the properties 1), and 3)-6) of Theorem 4.8 hold with the range of integration restricted to $M$. Indeed the proof of this result can be easily obtained by Theorem 4.8 setting

$$
U(x):= \begin{cases}u(x) & \text { if } x \in M, \\ \inf u & \text { if } x \in \mathbb{R}^{n} \backslash M,\end{cases}
$$

and therefore

$$
U^{*}(x):=\left\{\begin{aligned}
u^{*}(x) & \text { if } x \in M \\
\text { inf } u & \text { if } x \in \mathbb{R}^{n} \backslash M
\end{aligned}\right.
$$


We conclude this subsection with the following property, which is easy to prove but it is crucial for the next section.

Let $u$ be a function belonging to $C\left(\mathbb{R}^{n}\right)$. Denote its modulus of continuity by

$$
\omega_{u}(t):=\sup \{|u(x)-u(y)|:|x-y|<t\}, \quad t>0 .
$$

Notice that $u$ is uniformly continuous if and only if $\lim _{t \searrow_{0}} \omega_{u}(t)=0$.

Proposition 4.11. Let $u \in C\left(\mathbb{R}^{n}\right) \cap \mathcal{F}^{n}$. Then $u^{*} \in C\left(\mathbb{R}^{n}\right)$ and

$$
\omega_{u}(t) \geq \omega_{u^{*}}(t) \quad \forall t>0 .
$$

In particular, if $u$ is Lipschitz continuous with constant L, then also $u^{*}$ is Lipschitz continuous with a Lipschitz constant $L^{*}$ such that $L^{*} \leq L$.

Proof. By Remark 4.6 and (4.6) of Corollary 4.3, since $u$ is continuous we have that

$$
\operatorname{dist}\{\{u>t\} ; \partial\{u>s\}\} \leq \operatorname{dist}\left\{\left\{u^{*}>t\right\} ; \partial\left\{u^{*}>s\right\}\right\} \forall s, t \in \mathbb{R} \text { with } s<t,
$$

which implies (4.33). Since $L=\sup \left\{\omega_{u}(t) / t: t>0\right\}$, and similarly for $u^{*}$, the second assertion follows, too.

\subsection{Integral inequalities in Sobolev spaces}

In this subsection we state integral inequalities which involve derivatives of a function and its rearrangement. Variants of them are well known for different types of rearrangements, including Steiner symmetrization (see, for instance, [20], [11], and [16]), and they are usually referred to as Pólya-Szegö type inequalities. Theorem 4.12 below can be found as Theorem 6.1 and Corollary 6.1 in [6], and its proof is therefore omitted.

Theorem 4.12 (Pólya-Szegö's principle). Let $G=G\left(y, v, x^{\prime}\right)$ be a function belonging to $L^{\infty}\left(\mathbb{R}^{n} \times \mathbb{R}_{0}^{+} \times \mathbb{R}^{n-1}\right)$ where $y=\left(y_{1}, \ldots, y_{n}\right) \in \mathbb{R}^{n}$. Assume also that $G$ is continuous in $v$, convex in $y$, even in $y_{1}$ and nondecreasing in $y_{1}$ with $y_{1}>0$. Moreover let $u \in L^{\infty}\left(\mathbb{R}^{n}\right)$ be a nonnegative Lipschitz continuous function with compact support. Then

$$
\int_{\mathbb{R}^{n}} G\left(\nabla u, u, x^{\prime}\right) d \mu \geq \int_{\mathbb{R}^{n}} G\left(\nabla u^{*}, u^{*}, x^{\prime}\right) d \mu .
$$

Moreover, if $u \in W_{+}^{1, p}\left(\mu, \mathbb{R}^{n}\right)$, for some $p \in[1, \infty)$, then $u^{*} \in W_{+}^{1, p}\left(\mu, \mathbb{R}^{n}\right)$, and the inequality (4.35) holds if

$$
\left|G\left(y, v, x^{\prime}\right)\right| \leq C|y|^{p} \quad \text { for some } C>0,
$$

for any $\left(y, v, x^{\prime}\right) \in \mathbb{R}^{n} \times \mathbb{R} \times \mathbb{R}^{n-1}$. Finally, if $\Omega$ is a bounded domain in $\mathbb{R}^{n}$ and $u$ is a nonnegative function belonging to $W_{0}^{1, p}(\mu, \Omega)$ for some $p \in[1, \infty)$, then $u^{*} \in W_{0}^{1, p}\left(\mu, \Omega^{*}\right)$. 
Using Theorem 4.4 and proceeding analogously to the proof of Theorem 5.4 in [6], one can obtain a criterion for the equality case in the inequality (4.35). We omit the proof.

Theorem 4.13. Let $u \in W^{1, p}\left(\varphi, \mathbb{R}^{n}\right)$ for some $p \in[1, \infty)$. Furthermore, let the function $J:=\psi\left(\Psi^{-1}\right)$ be strictly convex, let $G \in C\left(\mathbb{R}^{n}\right), G=G(y), y=$ $\left(y_{1}, \ldots, y_{n}\right)$, let $G$ be convex and strictly increasing in $y_{1}$ for $y_{1}>0$, and such that

$$
|G(y)| \leq C\left(1+|y|^{p}\right), \quad \text { for some } C>0 .
$$

Finally, assume that

$$
\int_{\mathbb{R}^{n}} G(\nabla u) d \mu=\int_{\mathbb{R}^{n}} G\left(\nabla u^{*}\right) d \mu
$$

Then $u=u^{*}$.

\section{Radial measures}

In this section we consider measures $\mu$ whose density is a radially symmetric function, i.e.,

$$
d \mu=\varphi(|x|) d x
$$

where $\varphi \in C\left(\mathbb{R}_{0}^{+}\right)$is positive.

We prove isoperimetric inequalities with respect to special measures whose densities are

$$
\varphi_{1}(|x|)=\exp \left\{c|x|^{2}\right\}, \quad c>0, \quad \varphi_{2}(|x|)=|x|^{1-n} \exp \{a(|x|)\},
$$

where $a \in C\left(\mathbb{R}_{0}^{+}\right)$is convex.

If $M \in \mathcal{M}^{n}$, and if $\mu(M)<+\infty$, then let $M^{\star}$ denote the ball $B_{R}$ such that $\mu(M)=\mu\left(B_{R}\right)$. If $\mu(M)=+\infty$ then let $M^{\star}=\mathbb{R}^{n}$. We call $M^{\star}$ the Schwarz $\mu$-symmetrization of $M$. As in previous sections, we replace this definition by pointwise ones for open and closed sets. Thus, if $M$ is open/closed with finite $\mu$-measure, then let $M^{\star}$ be the open/closed ball centered at zero, having the same measure as $M$.

We ask for additional conditions on the measure $\mu$ such that the following isoperimetric inequality holds:

$$
P_{\mu}(M) \geq P_{\mu}\left(M^{\star}\right), \quad \forall M \in \mathcal{M}^{n},
$$

with equality if and only if $M=M^{\star}$. Although we are not able to give a necessary and sufficient condition for (5.2) to hold, we show below that the above isoperimetric property holds if

$$
d \mu=\exp \left\{c|x|^{2}\right\} d x, \quad c>0
$$

or if

$$
d \mu=|x|^{1-n} \exp \{a(|x|)\} d x, \quad \text { where } a \in C\left(\mathbb{R}_{0}^{+}\right) \text {is convex. }
$$


These isoperimetric inequalities are proved in Corollary 5.2 and Proposition 5.8, respectively.

Theorem 5.1. Let $\mu$ be the measure defined by (5.1) with $\varphi$ defined by (5.3). Then

$$
M^{\star}+B_{r} \subset\left(M+B_{r}\right)^{\star}, \quad \forall \text { Borel sets and } \forall r>0,
$$

and (5.2) holds. Furthermore, if $M$ and $N$ are open sets with $M \subset N$, then

$$
\operatorname{dist}\{M ; \partial N\} \leq \operatorname{dist}\left\{M^{\star} ; \partial N^{\star}\right\} \text {. }
$$

Finally, if $P_{\mu}(M)=P_{\mu}\left(M^{\star}\right)$ for some $M \in \mathcal{M}^{n}$, then $M=M^{\star}$.

Proof. Let $M$ be compact, $M \subset \overline{B_{R}}$ for some $R>0$, and set

$$
\begin{aligned}
A(M):=\left\{N \subset \mathbb{R}^{n}: N\right. \text { compact, } & N \subset \overline{B_{R}}, \mu(N)=\mu(M), \\
& \left.\mu\left(N+B_{r}\right) \leq \mu\left(M+B_{r}\right) \quad \forall r>0\right\} .
\end{aligned}
$$

Letting

$$
\delta:=\inf \left\{\mu\left(N \Delta M^{\star}\right): N \in A(M)\right\},
$$

there exists a sequence $\left\{N_{k}\right\} \subset A(M)$ with

$$
\lim _{k \rightarrow \infty} \mu\left(N_{k} \Delta M^{\star}\right)=\delta .
$$

Since the $N_{k}$ 's are equibounded, there is a subsequence $\left\{N_{k^{\prime}}\right\}$ which converges in Hausdorff distance to some set $N$, which also implies that $N \in A(M)$ and $\mu\left(N \Delta M^{\star}\right)=\delta$. Assume that $\delta>0$. Then we find two density points $\eta$ and $\zeta$ of $N$ and $M^{\star}$ such that $\eta \in M^{\star} \backslash N$ and $\zeta \in N \backslash M^{\star}$. After a rotation of the coordinate system

$$
x=\rho(\xi), \quad\left(x, \xi \in \mathbb{R}^{n}\right),
$$

we have

$$
\rho(\eta)=y=\left(y_{1}, y^{\prime}\right), \quad \rho(\zeta)=z=\left(z_{1}, y^{\prime}\right),
$$

for some $y^{\prime} \in \mathbb{R}^{n-1}, y_{1}, z_{1} \in \mathbb{R}$. Defining $N^{\prime}$ by

$$
N^{\prime}:=\rho(N) \equiv\{x=\rho(\xi): \xi \in N\}
$$

let $\left(N^{\prime}\right)^{*}$ denote its Steiner $\mu$-symmetrization w.r.t. the variable $x_{1}$. Notice that $\mu\left(N^{\prime} \Delta M^{\star}\right)=\delta$, and, due to the product structure of $\varphi$, we have that $\left(N^{\prime}\right)^{\star}=$ $\left(\left(N^{\prime}\right)^{*}\right)^{\star}=N^{\star}$, and $N^{\prime},\left(N^{\prime}\right)^{*} \in A(M)$. Since the slices $\left(N^{\prime}\left(y^{\prime}\right)\right)^{*}$ and $M^{\star}\left(y^{\prime}\right)$ are intervals centered at zero, it is easy to see that

$$
\mu_{1}\left(\left(N^{\prime}\left(y^{\prime}\right)\right)^{*} \cap M^{\star}\left(y^{\prime}\right)\right)>\mu_{1}\left(N^{\prime}\left(y^{\prime}\right) \cap M^{\star}\left(y^{\prime}\right)\right) .
$$

This also implies

$$
\mu\left(\left(N^{\prime}\right)^{*} \cap M^{\star}\right)>\mu\left(N^{\prime} \cap M^{\star}\right)
$$

and thus

$$
\mu\left(\left(N^{\prime}\right)^{*} \Delta M^{\star}\right)<\mu\left(N^{\prime} \Delta M^{\star}\right)
$$


contradicting the minimality of $\delta$. Hence $N=M^{\star}$, and (5.2) is proved for compact sets. It is easy to see that this also implies property (5.5) for compact sets, and by a simple approximation argument, as well for Borel sets. It is well known that (5.5) also implies (5.6), and the lower semicontinuity of the perimeter $P_{\mu}$ yields (5.2).

Assume finally that $P_{\mu}(M)=P_{\mu}\left(M^{\star}\right)$ for some $M \in \mathcal{M}^{n}$. Let $\rho$ be any rotation about the origin, and let ${ }^{*}$ denote $\mu$-Steiner symmetrization in direction $x_{1}$. Then $\left[(\rho M)^{*}\right]^{\star}=M^{\star}$, which means that $P_{\mu}(\rho M)=P_{\mu}\left((\rho M)^{*}\right)$. By Theorem 4.4 this implies that $\rho M=(\rho M)^{*}$. Since $\rho$ was arbitrary we have proved that $M$ is symmetric w.r.t. every $(n-1)$-hyperplane through the origin. Hence $M=M^{\star}$.

Next we rewrite the isoperimetric inequality in terms of $\mu(M)$. Let $I_{\mu}(m)$ be the isoperimetric function defined in (1.2), and define

$$
\begin{aligned}
h(r) & :=n \omega_{n} e^{c r^{2}} r^{n-1} \text { and } \\
H(r) & :=\int_{0}^{r} h(t) d t .
\end{aligned}
$$

Then

$$
P_{\mu}\left(M^{*}\right)=h\left(H^{-1}(\mu(M))\right)=I\left(\mu\left(M^{*}\right)\right) .
$$

Therefore (5.2) reads as follows:

Corollary 5.2. If $\mu(M)<+\infty$, then

$$
P_{\mu}(M) \geq h\left(H^{-1}(\mu(M))\right)=I\left(\mu\left(M^{*}\right)\right) .
$$

Now let us define the $\mu$-Schwarz symmetrization of functions with respect to the measure $\mu$ defined in (5.1) with $\varphi$ defined by (5.3). First we introduce a function $\tilde{u}:(0,+\infty) \rightarrow \mathbb{R}$ defined by

$$
\tilde{u}(s)=\inf \left\{t \in \mathbb{R}: m_{u}(t) \leq s\right\} .
$$

Notice that $\tilde{u}$ is a nonincreasing and right-continuous function. Observe also that $\tilde{u}(s)$ is the inverse function of $m_{u}(t)$, if $\tilde{u}(s)$ is not constant on intervals. In this case, the following equality holds:

$$
\frac{\partial \tilde{u}(s)}{\partial s}=\left[\frac{\partial m_{u}(t)}{\partial t}\right]^{-1}
$$

where $s=m_{u}(t)$.

If $u \in \mathcal{F}^{n}$ we define the Schwarz $\mu$-symmetrization of $u$ by

$$
u^{\star}(x):=\sup \left\{t \in \mathbb{R}: x \in\{u>t\}^{\star}\right\}, \quad x \in \mathbb{R}^{n} .
$$

Observe that, by definition of $\tilde{u}$ and $u^{\star}$, one has

$$
u^{\star}(x)=\tilde{u}(H(|x|)), \quad \text { for a.e. } x \in \Omega^{\star} .
$$

By definition $u^{\star}$ is radially symmetric and radially decreasing. Moreover $u$ and $u^{\star}$ are equimeasurable functions. If $u$ is continuous then we will understand this definition in pointwise sense, so that $u^{\star}$ is continuous, too. The assertions of Theorem 4.8 hold as well for the Schwarz $\mu$-symmetrization. 
As in the case of the Steiner $\mu$-symmetrization, the isoperimetric property (5.6) implies the following estimate for the modulus of continuity.

Proposition 5.3. Let $\mu$ be given by (5.1) and (5.3). Then

$$
\omega_{u} \geq \omega_{u^{\star}} \quad \forall u \in C\left(\mathbb{R}^{n}\right) \cap L^{\infty}\left(\mathbb{R}^{n}\right) \cap \mathcal{F}^{n} .
$$

The isoperimetric property (5.2) and a result due to Talenti [33], imply some Pólya-Szegö type inequalities, that is, integrals involving gradients of a nonnegative Lipschitz function having compact support decrease under weighted Schwarz symmetrization. The fact that the equality case in these inequalities occurs only in symmetric situations can be shown analogously as in [6], and by using Theorem 4.13 above. Using arguments as in the proofs of Theorem 4.8 and Corollary 4.3 this leads to norm inequalities in $W^{1, p}\left(\mathbb{R}^{n}\right)$. We omit the proofs.

Theorem 5.4. Let $u$ be a nonnegative Lipschitz continuous function on $\mathbb{R}^{n}$ with compact support, and let $G \in C\left(\mathbb{R}_{0}^{+}\right)$be nonnegative and convex with $G(0)=0$. Then

$$
\int_{\mathbb{R}^{n}} G(|\nabla u|) d \mu \geq \int_{\mathbb{R}^{n}} G\left(\left|\nabla u^{\star}\right|\right) d \mu .
$$

Moreover, if $G$ is strictly convex then (5.13) holds with equality only if $u=u^{\star}$. Furthermore, if $u \in W^{1, p}\left(\mu, \mathbb{R}^{n}\right)$ is nonnegative, for some $p \in[1, \infty)$, then $u^{\star} \in$ $W^{1, p}\left(\mu, \mathbb{R}^{n}\right)$, and (5.13) holds with $G(t)=t^{p}$. Finally, if $\Omega$ is a domain in $\mathbb{R}^{n}$ and $u \in W_{0}^{1, p}(\mu, \Omega)$ is nonnegative, then $u^{\star} \in W_{0}^{1, p}\left(\mu, \Omega^{\star}\right)$.

The isoperimetric inequality leads to several integral inequalities which compare an $L^{p}$-weighted norm of the gradient of a function with an $L^{q}$-weighted norm of the same function when the measure $\mu$ is given by (5.1) and (5.3). This type of result is also proved in a different way in [25].

Theorem 5.5. There are constants $C=C(p, q)>0$ such that for every $u \in C_{0}^{\infty}\left(\mathbb{R}^{n}\right)$,

$$
\|\nabla u\|_{p} \geq C(p, q)\|u\|_{q},
$$

where $q \in[p, n p /(n-p)]$ for $p \in[1, n), q \in[n,+\infty)$ for $p=n$, and $q \in[p,+\infty]$ for $p \in(n,+\infty)$. Moreover, there are constants $C(p)>0$ such that for every $u \in C_{0}^{\infty}\left(\mathbb{R}^{n}\right)$,

$$
\|\nabla u\|_{p} \geq C(p)\|u\|_{C^{0,1-(n / p)}\left(\mathbb{R}^{n}\right)},
$$

if $p \in(n,+\infty)$.

Proof. 1) Let $h$ and $H$ be given by (5.7) and (5.8). It is then easy to see that

$$
h(r)^{q} \geq C H(r) \quad \forall r \in[0,+\infty), \quad \text { for some } C>0,
$$

if $q \in[1, n /(n-1)]$. Applying Theorem 2.1.1 of [24], this implies (5.14) with $p=1$ and $q \in[1, n /(n-1)]$. 
2) Let $p>1, t>0, q \in[1, n /(n-1)], q<p /(p-1)$, and $u \in C_{0}^{\infty}\left(\mathbb{R}^{n}\right)$. Applying 1) and Hölder's inequality we have that

$$
\left\|u|u|^{t-1}\right\|_{q} \leq C(1, q)\left\|\left.t|u|^{t-1}\left|\nabla u\left\|_{1} \leq C_{0}\right\| \nabla u\left\|_{p}\right\|\right| u\right|^{t-1}\right\|_{p^{\prime}},
$$

for some $C_{0}>0$, where $p^{\prime}=p /(p-1)$. Choosing $t=p^{\prime} /\left(p^{\prime}-q\right)$, we obtain (5.14) for $p \in(1, n)$ with $q \in[p, n p /(n-p)]$, and for $p \geq n$ with $q \in[n,+\infty)$.

3) Since $\varphi(t)=\exp \left\{c t^{2}\right\} \geq 1$, there results

$$
\|\nabla u\|_{p} \geq\left(\int_{\mathbb{R}^{n}}|\nabla u|^{p} d x\right)^{1 / p},
$$

we obtain (5.15) from Morrey's Imbedding Theorem. From this we also obtain (5.14) for $p \in[n,+\infty)$ with $q=+\infty$.

Let $X^{p, q}$ denote the closure of $C_{0}^{\infty}\left(\mathbb{R}^{n}\right)$ with respect to the norm

$$
\mid\|u\|_{p, q}:=\|u\|_{q}+\|\nabla u\|_{p}, \quad p, q \in[1,+\infty) .
$$

From (5.14) one immediately obtains the following results.

Corollary 5.6. 1) Let $p \in[1,+\infty), q \in[p, n p /(n-p)]$ for $p<n$, and $q \in[p,+\infty)$ for $p \geq n$. Then

$$
X^{p, q}=W^{1, p}\left(\mathbb{R}^{n}, \varphi\right) .
$$

2) Let $\Omega$ be any domain in $\mathbb{R}^{n}$ and let $p \in[1,+\infty)$. Then $W_{0}^{1, p}(\Omega, \varphi) \subset$ $W^{1, p}\left(\mathbb{R}^{n}, \varphi\right)$, and

$$
\|\nabla u\|_{p, \Omega} \geq C(p, p)\|u\|_{p, \Omega} \quad \forall u \in W_{0}^{1, p}(\Omega, \varphi) .
$$

To our knowledge, the problem of finding the best constants in the inequalities (5.14) is still open. Here we solve such a problem in the special case $p=q=2$ :

Corollary 5.7. Let $c>0$. Then there holds

$$
\inf \left\{\frac{\|\nabla u\|_{2}^{2}}{\|u\|_{2}^{2}}: u \in C_{0}^{\infty}\left(\mathbb{R}^{n}\right)\right\}=2 c n=\frac{\left\|\nabla\left(e^{-c|x|^{2}}\right)\right\|_{2}^{2}}{\left\|e^{-c|x|^{2}}\right\|_{2}^{2}} .
$$

Proof. Consider the following eigenvalue problem for the harmonic oscillator:

$$
-\Delta v+c^{2}|x|^{2} v=\lambda v \quad \text { in } \mathbb{R}^{n} .
$$

The spectrum and the eigenfunctions are explicitly known (see [34], p.104 ff.). In particular, the spectrum is given by $\left\{\lambda=\lambda_{k}:(2 k-2+n) c, k=1,2, \ldots\right\}$, the eigenvalue $\lambda_{1}=c n$ is simple, and a corresponding eigenfunction is $v_{1}=\exp \left\{-c|x|^{2} / 2\right\}$. This implies

$$
\begin{aligned}
& \int_{\mathbb{R}^{n}}\left(|\nabla v|^{2}+c^{2}|x|^{2}|v|^{2}-c n|v|^{2}\right) d x \geq 0 \quad \forall v \in C_{0}^{\infty}\left(\mathbb{R}^{n}\right) \\
& \int_{\mathbb{R}^{n}}\left(\left|\nabla\left(\exp \left\{-c|x|^{2} / 2\right\}\right)\right|^{2}+c^{2}|x|^{2} \exp \left\{-c|x|^{2}\right\}-c n \exp \left\{-c|x|^{2}\right\}\right) d x=0
\end{aligned}
$$


Now let $u \in C_{0}^{\infty}\left(\mathbb{R}^{n}\right)$. Setting $v:=u \exp \left\{c|x|^{2} / 2\right\}$ we then find, by partial integration,

$$
\|\nabla u\|_{2}^{2}-2 c n\|u\|_{2}^{2}=\int_{\mathbb{R}^{n}}\left(|\nabla v|^{2}+c^{2}|x|^{2}|v|^{2}-c n|v|^{2}\right) d x,
$$

and the assertion follows from (5.22).

Finally we prove an isoperimetric inequality w.r.t. the measure $\mu$ given by

$$
d \mu=|x|^{1-n} \exp \{a(|x|)\} d x,
$$

where $a \in C\left(\mathbb{R}_{0}^{+}\right)$is convex (see [21] and [19]).

Notice that the measure $\mu$ above is singular at the origin. This implies in particular that the outer Minkowski content of the set $\{0\}$ is positive, namely

$$
\mu^{+}(\{0\})=n \omega_{n} e^{a(0)} \quad\left(\omega_{n} \text { is the measure of the } n \text {-dimensional unit ball }\right) .
$$

Proposition 5.8. Let $\Omega$ be a smooth open set in $\mathbb{R}^{n}$ which contains an open neighborhood of the origin, and such that $\mu(\Omega)<\infty$, where the measure $\mu$ is given by (5.24), and let $B_{R}$ be the ball with $\mu\left(B_{R}\right)=\mu(\Omega),(R>0)$. Then

$$
\mu^{+}(\Omega)=\int_{\partial \Omega}|x|^{1-n} \exp \{a(|x|)\} \mathcal{H}^{n-1}(d x) \geq n \omega_{n} e^{a(R)}=\mu^{+}\left(B_{R}\right) .
$$

Proof. Denote by $\nu$ the exterior normal to $\Omega$. By Green's Theorem, since the function $a$ is convex (see Lemma 6.4 in [21]), we have

$$
\begin{aligned}
\mu^{+}(\Omega) & =\int_{\partial \Omega}|x|^{1-n} \exp \{a(|x|)\} \mathcal{H}^{n-1}(d x) \geq \int_{\partial \Omega}|x|^{-n} \exp \{a(|x|)\}(x \cdot \nu) \mathcal{H}^{n-1}(d x) \\
& =n \omega_{n} e^{a(0)}+\int_{\Omega} \operatorname{div}\left(|x|^{-n} \exp \{a(|x|)\} x\right) d x \\
& =n \omega_{n} e^{a(0)}+\int_{\Omega} a^{\prime}(|x|) d \mu \geq n \omega_{n} e^{a(0)}+\int_{B_{R}} a^{\prime}(|x|) d \mu \\
& =\int_{\partial B_{R}}|x|^{-n} \exp \{a(|x|)\}(x \cdot \nu) \mathcal{H}^{n-1}(d x)=\mu^{+}\left(B_{R}\right) .
\end{aligned}
$$

Analogously to Theorem 5.4, the following result holds:

Theorem 5.9. Let $u$ be a nonnegative Lipschitz continuous function with compact support, and suppose that

$$
u(0)=\operatorname{ess} \sup u \text {. }
$$

Then (5.13) holds.

Proof. The proof can be carried out in a way analogous to the proof of Theorem 5.1 in [6], taking into account that the superlevel sets $\{x: u(x)>t\},(\sup u>t>0)$, contain an open neighborhood of the origin. We leave the details to the reader. 


\section{Comparison results}

In this section we assume that $\mu$ is the measure defined by (5.1) with $\varphi$ given by (5.3). We will prove a comparison result for weak solutions $u$ to nonlinear elliptic problems. This implies an estimate of the Schwarz $\mu$-symmetrization in terms of the solution of a related radially symmetric problem. We mention that similar results for the classical Schwarz symmetrization are well known (see, for instance, [2], [3], [30]). We also mention that a related comparison theorem holds for finite measure (see [6]) or for the symmetrization in Gauss space (see [5], [14]).

Consider the following nonlinear elliptic problem:

$$
\left\{\begin{aligned}
-\operatorname{div}(a(x, u, \nabla u)) & =f \varphi & & \text { in } \Omega \\
u & =0 & & \text { on } \partial \Omega .
\end{aligned}\right.
$$

Here $\Omega$ is an open subset of $\mathbb{R}^{N}, N \geq 2, p$ is a real number with $1<p<N$, and $a: \Omega \times \mathbb{R} \times \mathbb{R}^{N} \rightarrow \mathbb{R}^{N}$ is a Carathéodory function satisfying

$$
\begin{gathered}
a(x, s, \xi) \xi \geq \varphi(|x|)|\xi|^{p} \\
|a(x, s, \xi)| \leq \varphi(|x|)\left[|\xi|^{p-1}+|s|^{p-1}+a_{0}(x)\right], \quad a_{0}(x) \in L^{p^{\prime}}(\mu, \Omega), a_{0} \geq 0 \\
(a(x, s, \xi)-a(x, s, \eta), \xi-\eta)>0, \quad \xi \neq \eta,
\end{gathered}
$$

for almost every $x \in \Omega$ and for every $s \in \mathbb{R}, \xi \in \mathbb{R}^{N}, \eta \in \mathbb{R}^{N}$. Moreover we assume that

$$
f \in L^{\left(p^{*}\right)^{\prime}}(\varphi, \Omega)
$$

We will say that $u \in W_{0}^{1, p}(\Omega, \varphi)$ is a weak solution to the problem (6.1) if it satisfies

$$
\int_{\Omega} a(x, u, \nabla u) \nabla \psi d x=\int_{\Omega} f \psi \varphi d x, \quad \forall \psi \in W_{0}^{1, p}(\Omega, \varphi) .
$$

The existence of a weak solution is a consequence of the Sobolev type inequality given by Theorem 5.5 and an adaptation of classical results due to J. Leray and J.-L. Lions (cf. [23]).

The main result of this section is the following:

Theorem 6.1. Let $u$ be a weak solution to the problem (6.1). Denote by $v=v^{\star}(|x|)$ $\in W_{0}^{1, p}\left(\Omega^{\star}, \varphi\right)$ the function defined by

$$
v(|x|)=\int_{H(|x|)}^{\mu\left(\Omega^{\star}\right)} \frac{1}{[I(r)]^{p^{\prime}}}\left(\int_{0}^{r} \tilde{f}(\sigma) d \sigma\right)^{1 /(p-1)} d r,
$$

which is a weak solution to the problem

$$
\left\{\begin{aligned}
-\operatorname{div}\left(\varphi(x)|\nabla u|^{p-2} \nabla u\right) & =f^{\star}(x) \varphi(x) & & \text { in } \Omega^{\star}, \\
v & =0 & & \text { on } \partial \Omega^{\star} .
\end{aligned}\right.
$$


Then, for a.e. $x \in \Omega^{\star}$,

$$
u^{\star}(x) \leq v^{\star}(x) .
$$

Moreover, for any $1 \leq q<p$, there holds

$$
\|\nabla u\|_{q} \leq\|\nabla v\|_{q} .
$$

Proof. Let $t \in[0, \operatorname{ess} \sup |u|)$, let $h>0$, and let $\psi_{h}$ be the function defined by

$$
\psi_{h}= \begin{cases}\operatorname{sign} u & \text { if }|u|>t+h, \\ \frac{u-t \operatorname{sign} u}{h} & \text { if } t<|u| \leq t+h, \\ 0 & \text { otherwise. }\end{cases}
$$

Since $u$ belongs to $W_{0}^{1, p}(\Omega, \varphi)$ the function $\psi_{h}$ can be considered as a test function in (6.6) and we have

$$
\int_{\Omega} a(x, u, \nabla u) \nabla \psi_{h} d x=\int_{\Omega} f \psi_{h} \varphi d x
$$

or, equivalently,

$$
\begin{aligned}
\frac{1}{h} \int_{t<|u| \leq t+h} a(x, u, & \nabla u) \nabla u d x \\
& =\int_{|u|>t+h} f \operatorname{sign} u \varphi d x+\frac{1}{h} \int_{t<|u| \leq t+h} f(u-t \operatorname{sign} u) \varphi d x .
\end{aligned}
$$

By using the ellipticity condition (6.2), Hardy's inequality, and by letting $h$ go to zero, we have that

$$
-\frac{d}{d t} \int_{|u|>t} \varphi(|x|)|\nabla u|^{p} d x \leq \int_{0}^{m_{u}(t)} \tilde{f}(\sigma) d \sigma .
$$

Moreover, by Hölder's inequality, we find

(6.11) $-\frac{d}{d t} \int_{|u|>t} \varphi(|x|)|\nabla u| d x \leq\left(-\frac{d}{d t} \int_{|u|>t} \varphi(|x|)|\nabla u|^{p} d x\right)^{1 / p}\left(-m_{u}^{\prime}(t)\right)^{1 / p^{\prime}}$.

On the other hand, from Federer's coarea formula (cf. [17]), we obtain

$$
-\frac{d}{d t} \int_{|u|>t} \varphi(|x|)|\nabla u| d x=\int_{|u|=t} \varphi(|x|) \mathcal{H}_{n-1}(d x) .
$$

Combining (6.10), (6.11) and (6.12), we deduce

$$
\left(\int_{|u|=t} \varphi(|x|) \mathcal{H}_{n-1}(d x)\right)^{p} \leq\left(-m_{u}^{\prime}(t)\right)^{p / p^{\prime}} \int_{0}^{m_{u}(t)} \tilde{f}(\sigma) d \sigma .
$$

Now we apply the isoperimetric inequality given by Corollary 5.2, that is

$$
\int_{u=t} \varphi(|x|) \mathcal{H}_{n-1}(d x) \geq h\left(H^{-1}\left(m_{u}(t)\right)\right)=I\left(m_{u}(t)\right) .
$$


Combining (6.13) and (6.14), we get

$$
-\frac{\left[I\left(m_{u}(t)\right)\right]^{p}}{\left(-m_{u}^{\prime}(t)\right)^{p / p^{\prime}}} \leq \int_{0}^{m_{u}(t)} \tilde{f}(\sigma) d \sigma, \quad t \geq \operatorname{essinf} u .
$$

This implies

$$
-\frac{d \tilde{u}}{d s} \leq \frac{1}{[I(s))]^{p \prime}}\left(\int_{0}^{s} \tilde{f}(r) d r\right)^{1 /(p-1)} .
$$

Now an integration between $s>0$ and $\mu\left(\Omega^{\star}\right)$ gives

$$
\tilde{u}(s) \leq \int_{s}^{\mu\left(\Omega^{\star}\right)} \frac{1}{[I(r))]^{p^{\prime}}}\left(\int_{0}^{r} \tilde{f}(\sigma) d \sigma\right)^{1 /(p-1)} d r .
$$

Choosing $s=H(|x|)>0$ this concludes the proof of (6.8).

By Hölder's inequality, we have for any $1 \leq q<p$,

$$
-\frac{d}{d t} \int_{|u|>t} \varphi(|x|)|\nabla u|^{q} d x \leq\left(-\frac{d}{d t} \int_{|u|>t} \varphi(|x|)|\nabla u|^{p} d x\right)^{q / p}\left(-\mu_{u}^{\prime}(t)\right)^{1-q / p} .
$$

Using (6.10) this leads to

$$
-\frac{d}{d t} \int_{|u|>t} \varphi(|x|)|\nabla u|^{q} d x \leq\left(\int_{0}^{\mu_{u}(t)} \tilde{f}(s) d s\right)^{q / p}\left(-\mu_{u}^{\prime}(t)\right)^{1-q / p} .
$$

Integrating between 0 and $+\infty$ then gives

$$
\int_{\Omega} \varphi(|x|)|\nabla u|^{q} d x \leq \int_{0}^{+\infty}\left[-\mu^{\prime}(t)\right]^{-q / p}\left(\int_{0}^{\mu_{u}(t)} \tilde{f}(s) d s\right)^{q / p}(-d \mu(t)),
$$

from which one has, by (6.15),

$$
\int_{\Omega} \varphi(|x|)|\nabla u|^{q} d x \leq \int_{0}^{+\infty}\left(\frac{1}{I(s)}\right)^{q /(p-1)}\left(\int_{0}^{s} f^{*}(r) d r\right)^{\frac{q}{p(p-1)}} d s .
$$

This is $(6.9)$.

Remark 6.2. We emphasize that the proof of the comparison result carries over to domains with infinite $\mu$-measure. Indeed, since the solutions $u$ and $v$ belong to weighted Sobolev spaces, their level sets $\{x \in \Omega:|u|>t\}$ and $\{x \in \Omega:|v|>t\}$ have finite measure and therefore we can apply the isoperimetric inequality $(5.9)$ to such sets. Notice that in this case one has to replace $\Omega^{\star}$ in the symmetrized problem (6.7) by $\mathbb{R}^{n}$.

\section{References}

[1] Alvino, A., Díaz, J. I., Lions, P. L. And Trombetti, G.: Elliptic equations and Steiner symmetrization. Comm. Pure Appl. Math. 49 (1996), no. 3, 217-236.

[2] Alvino, A., Lions, P. L. And Trombetti, G.: Comparison results for elliptic and parabolic equations via Schwarz symmetrization. Ann. Inst. H. Poincaré Anal. Non Linéaire 7 (1990), no. 2, 37-65. 
[3] Alvino, A. and Trombetti, G.: Su una classe di equazioni ellittiche degeneri. Ricerche Mat. 29 (1980), 193-212.

[4] Benguria, R. And Linde, L.: A second eigenvalue bound for the Dirichlet Schrödinger operator. Comm. Math. Phys. 267 (2006), no.3, 741-755.

[5] Betta, M.F., Brock, F., Mercaldo, A. and Posteraro, M. R.: A comparison result related to Gauss measure. C.R. Math. Acad. Sci. Paris 334 (2002), no. 6, 451-456.

[6] Betta, M.F., Brock, F., Mercaldo, A. and Posteraro, M.R.: Weighted isoperimetric inequalities on $\mathbb{R}^{n}$ and applications. Math. Nachr. 281 (2008), no. 4, 466-498.

[7] Borell, C.: The Brunn-Minkowski inequality in Gauss space. Invent. Math. 30 (1975), no. 2, 207-216.

[8] Borell, C.: The Ornstein-Uhlenbeck velocity process in backward time and isoperimetry. Preprint, Chalmers University of Technology, 1986-03/ISSN 0347-2809.

[9] Brock, F., Chiacchio, F. And Mercaldo, A.: A class of degenerate elliptic equations and a Dido's problem with respect to a measure. J. Math. Anal. Appl. 348 (2008), no. 1, 356-365.

[10] Burago, Yu. D. And Zalgaller, V.A.: Geometric inequalities. Grundlehren der Mathematischen Wissenschaften 285, Springer Series in Soviet Mathematics, Springer-Verlag, Berlin, 1988.

[11] Cianchi, A. And Fusco, N.: Steiner symmetric extremals in Pólya-Szegö type inequalities. Adv. Math. 203 (2006), no. 2, 673-728.

[12] Crowe, J. A., Rosenbloom, P. C. And Zweibel, J. A.: Rearrangements of functions. J. Funct. Anal. 66 (1986), no. 3, 432-438.

[13] Dahlberg, J., Dubbs, A., Newkirk, E. And Tran, H.: Isoperimetric regions in the plane with density $r^{p}$. New York J. Math. 16 (2010), 31-51.

[14] Di Blasio, G. And Feo, F.: Nonlinear elliptic equations and Gauss measure. Matematiche (Catania) 61 (2006), no. 2, 245-274.

[15] Ehrhard, A.: Éléments extremaux pour les inégalités de Brunn-Minkowski gaussiennes. Ann. Inst. H. Poincaré Probab. Statist. 22 (1986), no. 2, 149-168.

[16] Esposito, L. And Trombetti, C.: Steiner symmetrization: a weighted version of Pólya-Szegö principle. NoDEA Nonlinear Differential Equations Appl. 14 (2007), no. 1-2, 219-231.

[17] Federer, H.: Geometric measure theory. Grundlehren der Mathematischen Wissenschaften 153, Springer-Verlag, New York, 1969.

[18] Ferone, V. and Mercaldo, A.: A second order derivation formula for functions defined by integrals. C.R. Acad. Sci. Paris Sér. I Math. 326 (1998), no. 5, 549-554.

[19] Howe, S.: The log-convex density conjecture and vertical surface area in warped products. ArXiv: 1107.4402v1 [math.DG], 22 Jul 2011.

[20] Kawohl, B.: Rearrangements and convexity of level sets in PDE. Lecture Notes in Mathematics 1150, Springer-Verlag, Berlin, 1985.

[21] Kolesnikov, A. And Zhdanov, R.: On isoperimetric sets of radially symmetric measures. In Concentration, functional inequalities and isoperimetry, 123-154. Contemp. Math. 545, Amer. Math. Soc., Providence, RI, 2011.

[22] Ledoux, M.: Semigroup proofs of the isoperimetric inequality in Euclidean and Gauss space. Bull. Sci. Math. 118 (1994), no. 6, 485-510. 
[23] Leray, J. And Lions, J. L.: Quelques résultats de Višik sur les problèmes elliptiques non linéaires par les méthodes de Minty-Browder. Bull. Soc. Math. France 93 (1965), 97-107.

[24] Maz'JA, V. G.: Sobolev spaces. Springer Series in Soviet Mathematics. SpringerVerlag, Berlin, 1985.

[25] Murthy, M.K. V. and Stampacchia, G.: Boundary value problems for some degenerate-elliptic operators. Ann. Mat. Pura Appl. (4) 80 (1968), 1-122.

[26] Rakotoson, J. M. And Simon, B.: Relative rearrangement on a measure space. Application to the regularity of weighted monotone rearrangement. I. Appl. Math Lett. 6 (1993), no. 1, 75-78.

[27] Rakotoson, J. M. And Simon, B.: Relative rearrangement on a measure space. Application to the regularity of weighted monotone rearrangement. II. Appl. Math. Lett. 6 (1993), no. 1, 79-82.

[28] Rosales, C., Cañete, A., Bayle, V. and Morgan, F.: On the isoperimetric problem in Euclidean space with density. Calc. Var. Partial Differential Equations 31 (2008), no. 1, 27-46.

[29] Steiner, J.: Einfacher Beweis der isoperimetrischen Hauptsätze. Crelle J. Reine Angew. Math. 18 (1838), 281-296; Ges. Werke II, Berlin (1882), 77-91 (in German).

[30] Talenti, G.: Elliptic equations and rearrangements. Ann. Scuola Norm. Sup. Pisa Cl. Sci. (4) 3 (1976), no. 4, 697-718.

[31] Talenti, G.: Linear elliptic PDEs: level sets, rearrangements and a priori estimates of solutions. Boll. Un. Mat. Ital. B (6) 4 (1985), no. 3, 917-949.

[32] Talenti, G.: The standard isoperimetric theorem. In Handbook of convex geometry, Vol. A-B, 73-124. North-Holland, Amsterdam 1993.

[33] Talenti, G.: A weighted version of a rearrangement inequality. Ann. Univ. Ferrara Sez. VII (N.S.) 43 (1997), 121-133.

[34] Taylor, M. E.: Partial differential equations II. Qualitative studies of linear equations. Applied Mathematical Sciences 116, Springer, New York, 1996.

Received August 4, 2011.

Friedemann Brock: Institute of Mathematics, Leipzig University, Augustusplatz, 04109 Leipzig, Germany.

E-mail: brock@math.uni-leipzig.de

Anna Mercaldo: Dipartimento di Matematica e Applicazioni "R. Caccioppoli", Università degli Studi di Napoli "Federico II", Complesso Monte S. Angelo, via Cintia, 80126 Napoli, Italy.

E-mail: mercaldo@unina.it

Maria Rosaria Posteraro: Dipartimento di Matematica e Applicazioni "R. Caccioppoli", Università degli Studi diNapoli "Federico II", Complesso Monte S. Angelo, via Cintia, 80126 Napoli, Italy.

E-mail: posterar@unina.it 